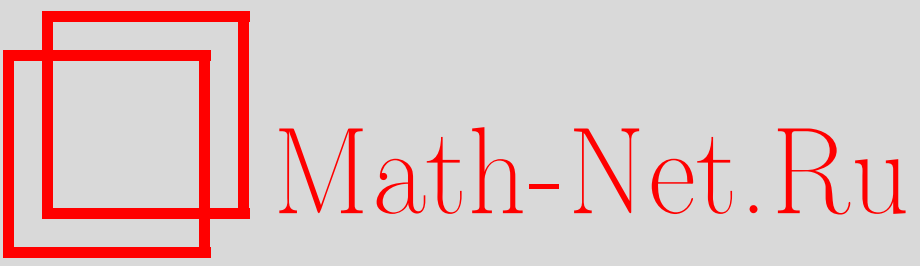

О. Д. Аносова, Инвариантные многообразия и динамические бифуркации, $У M H$, 2005, том 60, выпуск 1, 157-158

DOI: https://doi.org/10.4213/rm1387

Использование Общероссийского математического портала Math-Net.Ru подразумевает, что вы прочитали и согласны с пользовательским соглашением

http://www.mathnet.ru/rus/agreement

Параметры загрузки:

IP : 54.196 .121 .252

26 апреля 2023 г., 17:23:09 


\title{
ИНВАРИАНТНЫЕ МНОГООБРАЗИЯ И ДИНАМИЧЕСКИЕ БИФУРКАЦИИ
}

\author{
О. Д. АносовА
}

Данная заметка посвящена исследованию сохранения экспоненциалшно устойчивого или гиперболического инвариантного многообразия при динамической бифуркации, т.е. при бифуркации в системе, зависящей от параметра, где параметр изменяется со временем.

1. Основные определения. Рассмотрим $C^{r}$-гладкое векторное поле $v$, определенное в некоторой области $V$ евклидова пространства $\mathbb{R}^{n}$. Все рассматриваемые далее многообразия считаются $C^{r}$-гладкими и компактными.

Пусть $M$ - многообразие с границей $\partial M$ (и, возможно, с углами), лежащее в области $V$. Многообразие $M$ называется растекающимся (или отрицательно инвариантным) для поля $v$, если поле $v$ касается $M$ во всех точках многообразия $M$ и во всех регулярных граничных точках

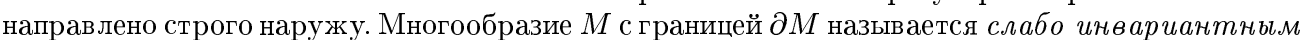
(или интегральным) многообразием для векторного поля $v$, если поле $v$ касается $M$ во всех точках многообразия $M$. Это означает, что траектория может как покинуть многообразие $M$, так и прийти в $M$, но только через его границу $\partial M$; также движение на границе может отсутствовать.

Обозначим через $g^{t}$ поток, задаваемый векторным полем $v$. Пусть $\pi_{a}: T_{a} \mathbb{R}^{n} \rightarrow N_{a} M$ - оператор ортогонального проектирования в точке $a \in M$ и $d g^{t}(a): T_{a} \mathbb{R}^{n} \rightarrow T_{g^{t}} a^{n}$ - диффференциал отображения $g^{t}$ в точке $a \in V$. Показателем притяжсения в точке а многообразия $M$ для векторного поля $v$ называется число

$$
\lambda_{N}(M, a)=\underset{t \rightarrow+\infty}{\lim _{\rightarrow}}-\frac{\ln \left\|\pi_{a} \circ d g^{t}\left(g^{-t} a\right)\right\|}{t} .
$$

Показателем сближения траекторий в точке а многообразия $M$ векторного поля $v$ называется число

$$
\lambda_{T}(M, a)=\varlimsup_{t \rightarrow+\infty} \frac{\ln \left\|\left.d g^{-t}(a)\right|_{T_{a} M}\right\|}{t} .
$$

Обозначения подчеркивают, что показатель $\lambda_{N}$ характеризует сжатие в направлении, нормальном к $M$, а показатель $\lambda_{T}$ - в касательном.

Динамическая бифуркачия - это бифуркация в системе, зависящей от параметра, в которой параметр в свою очередь изменяется со временем ("находится в динамике"). Точнее, каждая "обькновенная" бифуркация, задаваемая системой вида

$$
\dot{x}=v(x, y), \quad x \in \mathbb{R}^{n}, \quad y \in \mathbb{R}^{m},
$$

с малым многомерным параметром $y$ порождает динамическую бифуркацию, или, другими словами, быстро-медленную систему, вида

$$
\dot{x}=f_{1}(x, y, \varepsilon), \quad \dot{y}=\varepsilon f_{2}(x, y, \varepsilon),
$$

где $\varepsilon \in \mathbb{R}$ - малшй параметр и при $\varepsilon=0$ выполнено равенство $f_{1}(x, y, 0)=v(x, y)$.

2. Формулировка теоремы для устойчивого инвариантного многообразия. Предположим, что система $\dot{x}=f_{1}(x, 0,0)$, где $f_{1}(x, y, \varepsilon)-C^{r}$-гладкая функция, $x \in \mathbb{R}^{k}$, $y \in \mathbb{R}^{l}, \varepsilon \in \mathbb{R}$, имеет компактное $C^{r}$-гладкое либо замкнутое инвариантное, либо растекающееся многообразие $M_{0} \subset \mathbb{R}^{k}$ такое, что для того же $r \in \mathbb{N}$ в каждой точке $а \in M_{0}$ ляпуновские числа удовлетворяют неравенству $+\infty>\lambda_{N}\left(M_{0}, a\right)>$ $\max \left(0, r \lambda_{T}\left(M_{0}, a\right)\right)$. Тогда для любой $C^{r}$-гладкой функции $f_{2}$ существуют такие числа $\varepsilon>0$ и $y_{0}>0$, что для любого $y \in B_{y_{0}}:=\left\{y:|y|<y_{0}\right\} \subset \mathbb{R}^{l}$

1) при каждом $\varepsilon \in\left(0, \varepsilon_{0}\right]$ соответствующая динамическая бифуркачия, задаваемая системой (2), имеет $C^{r}$-гладкое слабо инвариантное многообразие $\mathfrak{M}_{\varepsilon} \subset$ $\mathbb{R}^{k} \times B_{y_{0}}, C^{r}$-близкое кмногообразию $\mathfrak{N}_{0}:=M_{0} \times B_{y_{0}}$

2) семейство $\left\{\mathfrak{M}_{\varepsilon}\right\} C^{r}$-гладко зависит от $\varepsilon$.

Работа выполнена при частичной финансовой поддержке Российского фонда фундаментальных исследований (грант № 02-01-22002). 
3. План доказательства. Ограничимся случаем растекающегося многообразия $M_{0}$. Возьмем вспомогательную двупараметрическую систему

$$
\dot{x}=f_{1}(x, z, \lambda \mu), \quad \dot{z}=\varphi(|z|) z+\mu f_{2}(x, z, \lambda \mu),
$$

где $x \in \mathbb{R}^{k}, z \in B_{z} \in \mathbb{R}^{l}, B_{z}$ - единичный шар, $\lambda, \mu \in \mathbb{R}$ - малые параметры, $\varphi(t)$ - неубывающая $C^{\infty}$-функция, равная 0 при $t \in[0,1-\delta]$ и равная $\delta>0$ при $t=1$. Проверяется, что при вьполнении условий теоремы система (3) при $\lambda=\mu=0$ имеет $C^{r}$-гладкое растекающееся многообразие $\mathfrak{M}_{0,0}=M_{0} \times B_{z}$, на котором выполняются условия на показатели из теоремы. Тогда можно применить теорему Феничеля о сохранении инвариантного многообразия [1; с. 205], согласно которой существуют такие числа $\lambda_{0}, \mu_{0}>0$, что система (3) при $\lambda \in\left[0, \lambda_{0}\right)$ и $\mu \in\left[0, \mu_{0}\right)$ имеет $C^{r}$-гладкое растекающееся многообразие $\mathfrak{M}_{\lambda, \mu}, C^{r}$-близкое к $\mathfrak{M}_{0,0}$.

Рассмотрим произвольное сечение $\lambda=\lambda_{1}<\lambda_{0}$. Заменой переменных $y=\lambda_{1} z, \varepsilon=\lambda_{1} \mu$ в области $z \in B_{z}^{1-\delta}=\{z:|z| \leqslant 1-\delta\}, y \in B_{y_{0}}=\left\{y:|y| \leqslant y_{0}=\lambda_{1}(1-\delta)\right\}$ система (3) приводится к исходной системе (2). Тогда многообразие $\mathfrak{M}_{\varepsilon}:=\mathfrak{M}_{\lambda, \mu} \cap\left\{z \in B_{z}^{1-\delta}\right\} \cap\left\{\lambda=\lambda_{1}\right\}$ определено при $y \in B_{y_{0}}, \varepsilon \in\left[0, \varepsilon_{0}\right], \varepsilon_{0}=\lambda_{1} \mu_{0}$, является слабо инвариантным относительно исходной системы (2) и $C^{r}$-гладким (как сечение $C^{r}$-гладкого многообразия $\mathfrak{M}_{\lambda, \mu}$ ). Слабая инвариантность возникает из-за уменьшения области определения по переменной $y$ в исходной системе (2) по сравнению с областью определения по переменной $z$ во вспомогательной системе (3).

Вторая часть теоремы доказьвается тем же способом с применением обобщения теоремы Феничеля из статьи [2].

4. Применение теоремы. Аналогичньй резултат верен для случая, когда исходная система имеет нормально гиперболическое инвариантное многообразие.

Задачи по систематической разработке теории динамических бифуркаций как развития теории "обыкновенных" бифуркаций были предложены Дж. Гукенхеймером [3]. Методы данной работы применимы, в частности, к исследованию динамических бифуркаций гомоклинических траекторий седлоузла и гомоклинических поверхностей седлоузлового цикла. (Обычные бифуркации для этих случаев, т.е. бифуркации при $\varepsilon=0$, исследованы в [4], [5]].) В перечисленных случаях при динамической бифуркации приведенная теорема позволяет выделить некоторое инвариантное многообразие относительно небольшой размерности.

Автор благодарит Ю. С. Ильяшенко и Д. В. Аносова за постановку задачи и полезные обсуждения.

\section{СПИСОК ЛИТЕРАТУРЫ}

[1] N. Fenichel // Indiana Univ. Math. J. 1971. V. 21. № 3. P. 193-226. [2] О. Д. Аносова // Труды МИАН. 2002. Т. 236. С. 27-32. [3] J. Guckenheimer // Nonlinear Dinamical Systems and Chaos (Groningen, 1995). Basel: Birkhäuser, 1996. P. 213-225. (Progr. Nonlinear Differential Equations Appl. V. 19.) [4] Ю. С. Ильяшенко, Л. Вейгу. Нелокальные бифуркации. М.: МЦНМО / ЧеРо, 1999. (Новые матем. дисциплины.) [5] А. Р. Борисюк // Матем. заметки. 2002. T. 71. № 3. C. 348-363.

Государственный университет -

Высшая школа экономики
Представлено Ю. С. Ильяшенко Принято редколлегией 28.01.2005 